\title{
Philosophiques
}

\section{L’horizon et le destin de la phénoménologie}

\section{Aurélien Djian}

Volume 45, numéro 2, automne 2018

URI : https://id.erudit.org/iderudit/1055267ar

DOI : https://doi.org/10.7202/1055267ar

Aller au sommaire du numéro

\section{Éditeur(s)}

Société de philosophie du Québec

\section{ISSN}

0316-2923 (imprimé)

1492-1391 (numérique)

Découvrir la revue

Citer cet article

Djian, A. (2018). L'horizon et le destin de la phénoménologie. Philosophiques, 45(2), 343-364. https://doi.org/10.7202/1055267ar

\section{Résumé de l'article}

L'ambition de cet article est double. Il s'agit d'abord de fixer le contexte philosophique qui sous-tend le débat entre Derrida et Marion en 1999, à l'Université de Villanova, et de réviser la perspective qui y est formulée selon laquelle le destin de la phénoménologie est intimement lié à une décision à prendre à l'égard du concept d'horizon : « il n’y a pas de phénoménologie sans horizon ", affirme Derrida à Marion, il faut donc la dépasser ; « la dernière étape en vue d'une véritable phénoménologie serait d'abandonner le concept d'horizon ", rétorque Marion à Derrida. Cette révision de perspective nous conduira alors, dans un second temps, à restituer, en se servant de l'histoire du concept d'horizon comme fil directeur heuristique, le sens de ce que nous appellerons l'histoire française de l'herméneutique phénoménologique, dont l'unité, par-delà la multiplicité des étiquettes, réside dans le partage d'une même définition de l'activité philosophique, comme tentative de laisser se montrer soi-même à partir de soi-même le phénomène par excellence, et, avec et contre le second Heidegger, d'une même exigence de dépassement de l'horizon, solidaire de la métaphysique moderne de la subjectivité. Le principe d'une telle reconstruction permettra enfin d'envisager la possibilité d'une histoire différenciée des concepts alternatifs de phénoménologie et d'horizon, qui reste à écrire.
Ce document est protégé par la loi sur le droit d'auteur. L'utilisation des services d’Érudit (y compris la reproduction) est assujettie à sa politique d'utilisation que vous pouvez consulter en ligne.

https://apropos.erudit.org/fr/usagers/politique-dutilisation/ 


\title{
L'horizon et le destin de la phénoménologie
}

\author{
AURÉLIEN DJIAN \\ Université de lille, umr/cnrs, stl 8163 \\ aurelien.djian@univ-lille.fr
}

\begin{abstract}
RÉSUMÉ. - L'ambition de cet article est double. Il s'agit d'abord de fixer le contexte philosophique qui sous-tend le débat entre Derrida et Marion en 1999, à l'Université de Villanova, et de réviser la perspective qui y est formulée selon laquelle le destin de la phénoménologie est intimement lié à une décision à prendre à l'égard du concept d'horizon: «il n'y a pas de phénoménologie sans horizon », affirme Derrida à Marion, il faut donc la dépasser; «la dernière étape en vue d'une véritable phénoménologie serait d'abandonner le concept d'horizon », rétorque Marion à Derrida. Cette révision de perspective nous conduira alors, dans un second temps, à restituer, en se servant de l'histoire du concept d'horizon comme fil directeur heuristique, le sens de ce que nous appellerons I'histoire française de l'herméneutique phénoménologique, dont l'unité, pardelà la multiplicité des étiquettes, réside dans le partage d'une même définition de l'activité philosophique, comme tentative de laisser se montrer soi-même à partir de soi-même le phénomène par excellence, et, avec et contre le second Heidegger, d'une même exigence de dépassement de l'horizon, solidaire de la métaphysique moderne de la subjectivité. Le principe d'une telle reconstruction permettra enfin d'envisager la possibilité d'une histoire différenciée des concepts alternatifs de phénoménologie et d'horizon, qui reste à écrire.
\end{abstract}

ABSTRACT. - The task of this article is twofold. First, it is meant to sketch out the philosophical context within which the Derrida/Marion debate that happened in 1999 at the university of Villanova took place, and to overhaul the perspective that it puts forward, that the destiny of phenomenology itself is linked at the abandon of the concept of horizon: "Without horizon there is no phenomenology", argues Derrida against Marion; "the last step for a real phenomenology would be to give up the concept of horizon", answers Marion to Derrida. This overhaul will then leads us to use the history of the concept of horizon as a heuristical guiding thread to flesh out what makes the unity of what we can call the French history of hermeneutical phenomenology, that is to say, firstly, the sharing of the same definition of philosophy, as the attempt to let the distinctive phenomenon be seen as it shows itself by itself. And, secondly, with and against the second Heidegger, of the same requirement of overcoming the horizon, as it is committed to the modern metaphysics of subjectivity. The principle on which such a reconstruction is based will finally allow us to consider the idea of a differentiated history of the alternative concepts of phenomenology and horizon. History which is yet to come.

DERRIDA: Dissocieriez-vous alors ce que vous appelez phénoménologie de l'autorité du "en tant que tel»? S'il en est ainsi, ce serait la première hérésie en phénoménologie. Une phénoménologie sans «en tant que tel»!

MARION: Pas la première, non! Il y en a d'autres. J'ai dit à Lévinas il y a quelques années qu'en fait, la dernière étape en vue d'une véritable phénoménologie serait 
d'abandonner le concept d'horizon. Levinas m'a répondu immédiatement: «sans horizon, il n’y a pas de phénoménologie». Et je soutiens témérairement qu'il avait tort.

DERRIDA: Je suis aussi en faveur d'une suspension de l'horizon mais, pour cette raison justement, en affirmant cela, je ne suis plus un phénoménologue. Je suis très fidèle à la phénoménologie, mais quand j’accepte la nécessité de suspendre l'horizon, je ne suis plus un phénoménologue.

(Jean-Luc Marion, On the gift: 66, I7).

\section{1. «Sans horizon, il n'y a pas de phénoménologie»}

Les remarques de Jean-Luc Marion et de Jacques Derrida sur l'horizon, au détour d'un débat sur le don qui eut lieu en I999, à l'Université de Villanova, esquissent un singulier changement de perspective qui fait voir autrement l'histoire familière de la phénoménologie d'inspiration husserlienne ${ }^{1}$. Nous avons en effet l'habitude de la regarder à la lumière d'autres concepts bien plus illustres, dont la signification pour le destin de cette phénoménologie est systématiquement mise en avant, renvoyant parfois aux origines de la philosophie elle-même, généralement employés en premier lieu par Husserl puis développés, critiqués, refondus, dépassés par ses successeurs - du phénomène à l'intentionnalité en passant par l'intuition catégoriale, l'a priori, la transcendance, le corps vivant, le sens, pour n'en rester ici qu'aux notions les plus en vue. Autrement dit, en dépit de l'importance reconnue du concept dans la tradition phénoménologique à partir du $\mathrm{XX}^{\mathrm{e}}$ siècle - chez Husserl2 Heidegger $^{3}$ et certains de ses élèves, particulièrement Gadamer ${ }^{4}$, chez MerleauPonty $^{5}$ et jusqu'à la tentative récente de Klaus Held d'une phénoménologie du monde politique (20IO) —, rien ne semble à première vue préparer cette notion à présider au destin de «la » phénoménologie elle-même: soit qu'elle décide de sa vie ou de sa mort — «sans horizon, il n’y a pas de phénoménologie» réplique Levinas à Marion, ou, sous sa forme derridienne, «quand j'accepte la nécessité de suspendre l'horizon, je ne suis plus un phénoménologue»; soit que son abandon, comme le soutient Marion, constitue «la der-

1. Nous employons l'expression de "phénoménologie d'inspiration husserlienne » pour la distinguer d'autres formes de phénoménologie qui ne sont pas directement concernées par le débat en question, celle de Lambert, de Hegel, de Brentano, ou encore la phénoménologie ou «phanéroscopie» de Peirce. Nous reviendrons brièvement sur la question des rapports entre ces phénoménologies dans la conclusion.

2. Voir notamment Romano (2010: 657-672), Blumenberg (2010: 89sq.), Geniusas (2OI2: 6-IO).

3. Voir Elm (2004).

4. Pour Gadamer et l'importance du concept de fusion des horizons, voir Deniau (20I 5 ) et Grondin (2005). Pour l'usage de l'horizon dans le mouvement d'inspiration gadamérienne de l'herméneutique littéraire, voir notamment Jauss (I967: I 85-6/65-7) et Iser (I976: I64/I 82).

5. Voir Merleau-Ponty (I945: 8I-6) et Schweidler (2004). 
nière étape en vue d'une véritable phénoménologie ", ou plus précisément l'ultime «hérésie » par rapport à «la » phénoménologie que, par contraste, on peut appeler «orthodoxe» - sans d'ailleurs qu'aucun des interlocuteurs ne précise ici de quelle phénoménologie il s'agit.

Or la première hypothèse de ce travail est que c'est précisément par la détermination de la forme spécifique de phénoménologie qui constitue le centre de gravitation de ce débat, et à laquelle il y est fait référence de manière indéterminée sous le titre de "la phénoménologie ", que s'éclaire à la fois la nature véritable de ce dernier, le problème spécifique de l'horizon qui lui revient et, enfin, la relation précise qu'entretiennent la phénoménologie et l'horizon dans ce contexte, c'est-à-dire dans le cadre de l'histoire d'une phénoménologie bien déterminée. Dans quelle mesure une telle enquête généalogique sur ce type de phénoménologie doit permettre de réviser la perspective exprimée dans le débat Derrida/Marion sur la double histoire entrelacée de «la» phénoménologie et de l'horizon ( «il n’y a pas de phénoménologie sans horizon» vs «la phénoménologie véritable est sans horizon»), c'est ce que nous allons voir dans un premier temps. Mais les résultats de cette enquête devront à leur tour nous permettre d'explorer une seconde hypothèse: s'il est vrai que l'histoire de cette phénoménologie est bien entrelacée, d'une manière tout à fait particulière et que nous allons déterminer, à celle d'un certain usage du concept d'horizon, on doit pouvoir se servir de l'histoire de ce dernier comme fil directeur pour esquisser le sens de ce que nous appellerons dans ce qui suit «l'histoire française de la phénoménologie» - l'expression étant prise ici dans un sens très spécifique que nous définirons plus loin. Ainsi, c'est à l'établissement d'un principe permettant de reconstruire un pan de l'histoire de la phénoménologie française en particulier, et, comme nous le verrons, de la phénoménologie d'inspiration heideggerienne en général, que nous espérerons avoir contribué à la fin de ce travail. Enfin, dans la conclusion, nous chercherons à élargir un peu plus la perspective et à discuter des développements possibles qu'ouvre un tel principe pour la réalisation d'une histoire différenciée des phénoménologies et des usages divers et variés de l'horizon.

\section{Généalogie de «la» phénoménologie»}

Si «la phénoménologie au sens large est la somme de l'œuvre husserlienne et des hérésies issues de Husserl» (Ricœur 1986: 9), le problème phénoménologique de l'abandon de l'horizon n'est pas une hérésie par rapport à l'orthodoxie husserlienne; au contraire, non seulement ce problème prend la forme pour les phénoménologues français d'une confrontation directe avec Heidegger et, à travers lui, avec l'ensemble de la tradition philosophique comme telle - l'orthodoxe n'est donc pas celui qu'on croit -, mais, plus profondément encore, il naît de l'évolution interne de la pensée heideggerienne elle-même - Heidegger est le premier hérétique de sa propre orthodoxie. C'est précisément ce que montre, et ce que masque, Totalité et Infini 
de Levinas, dans lequel ce dernier formule une thèse sur la phénoménologie qui constitue manifestement le point de référence commun du débat Derrida/Marion.

L'entreprise philosophique menée dans Totalité et Infini consiste à opposer à l'impérialisme ontologique qui domine la philosophie occidentale (TI: 6) - le fait transcendantal d'une certaine manière de penser philosophiquement l'être et autrui à partir de la catégorie de la totalité, dans laquelle Autrui est réduit par le biais «d'un terme moyen et neutre qui assure l'intelligence de l'être" $(T I: 34)$ à un autre moi, à l'égard duquel tout est permis - un rapport plus originaire à l'autre qui "conditionne la totalité ellemême» (TI: 9), et dans lequel celui-ci est considéré moralement, paisiblement, comme un autre que moi qui exige que je lui rende justice, en face duquel je me reconnais des «obligations» $(T I: 36)$. Or, s'il est vrai que toute la philosophie occidentale a pensé l'être à partir du concept de totalité, "la phénoménologie tout entière, depuis Husserl» $(T I: 35)$ ne fait pas exception à la règle. Elle constitue même un cas "où "l'impérialisme ontologique" est encore plus visible », dans la mesure où y est explicitement assumé que «la question fondamentale de la philosophie en général» est celle du sens de l'être (SZ: 27/42). Quant à l'horizon, il désigne cette nouvelle variante introduite par la phénoménologie — après la liberté socratique, le concept de l'idéalisme ou le vécu de l'affection de l'idéalisme berkeleyen (TI:34-5) — de l'intermédiaire par lequel l'Autre est réduit au Même:

[L]a médiation phénoménologique emprunte une autre voie [...]. C'est l'être de l'étant qui est le medium de la vérité. La vérité concernant l'étant suppose l'ouverture préalable de l'être [...]. L'étant se comprend dans la mesure où la pensée le transcende, pour le mesurer à l'horizon où il se profile. La phénoménologie tout entière, depuis Husserl, est la promotion de l'idée de l'horizon [...]; l'étant surgit sur un fond qui le dépasse comme l'individu à partir du concept $(T I: 35)$.

Deux choses sont ici fondamentales. D'abord, non seulement Levinas étend à la définition de la phénoménologie depuis Husserl une doctrine qui a en vérité été défendue dans une certaine phénoménologie, à savoir l'herméneutique phénoménologique d'Être et Temps - le Dasein est cet étant singulier entre tous qui comprend l'étant dans l'horizon de l'être, et l'être dans l'horizon du temps - , mais, en faisant la démonstration de son accointance avec le présupposé belliciste de la philosophie occidentale comme telle, qui s'exprime dans son subjectivisme - «Sein und Zeit n'a peut-être soutenu qu'une seule thèse: l'être est inséparable de la compréhension de l'être (qui se déroule comme temps), l'être est déjà appel à la subjectivité » $(T I: 36)$ - il généralise ipso facto la critique à l'ensemble de «la " phénoménologie ( «la phénoménologie tout entière, depuis Husserl...»), qui devient sans distinction la dernière variante en date du mode de pensée de l'être en vogue depuis la naissance de la philosophie occidentale, et par là à l'ensemble de la tradition occidentale elle-même. 
Ensuite, et c'est là que la thèse de Totalité et Infini semble se distinguer de la formule rapportée par Marion, Levinas oppose certes à l'impérialisme ontologique, et par conséquent à la phénoménologie depuis Husserl, l'idée d'une éthique comme philosophie première; mais il suggère également que cette éthique prend la forme d'une autre phénoménologie, comme il le rappelle dans la préface à l'édition allemande de janvier I 987 : «ce livre qui se veut et se sent d'inspiration phénoménologique procède d'une longue fréquentation des textes husserliens, et d'une incessante attention à Sein und Zeit» (TI: I). En ce sens, avant Marion, Levinas avait déjà indiqué la possibilité d'une autre phénoménologie sans horizon, autre par rapport à la lettre de la phénoménologie husserlienne, objectivante et subjectiviste, mais proche de l'esprit de cette dernière ${ }^{6}$. On pourrait même aller plus loin, et la présenter comme une phénoménologie se voulant d'inspiration husserlienne, et par conséquent fondée sur un autre concept d'horizon correspondant, aux yeux de Levinas, à l'esprit de celui, husserlien, d'horizon. C'est ce qu'atteste la remarque dans la préface, soulignant que «l'enseignement essentiel » de la notion husserlienne d'horizon est que toute pensée implique «des horizons insoupçonnés par cette pensée »-Autrui, en ce sens, se révèle dans son visage comme un horizon et manifeste le "débordement de la pensée objectivante par une structure oubliée dont elle vit» (TI: I4) —, horizons qui «dans la phénoménologie husserlienne, prise à la lettre", sont interprétés comme "pensées visant des objets" (ibid.).

Mais quoiqu'il en soit de ce dernier point, l'analyse que nous venons de proposer de Totalité et Infini permet déjà de commencer à clarifier la nature du débat Derrida/Marion, et le problème spécifique de l'horizon qui le sous-tend. En effet, l'opposition directe, contre l'orthodoxie heideggerienne représentée par Être et Temps et son modèle de l'horizon de la compréhension du Dasein, et indirecte, contre "la» phénoménologie comme telle par le biais de la généralisation du modèle heideggerien d'Être et Temps à l'ensemble de la phénoménologie depuis Husserl, et contre la tradition philosophique occidentale dans son ensemble, définit une croisée des chemins: si l'orthodoxie heideggerienne et son subjectivisme, exprimé aux yeux de Levinas dans le concept d'horizon qui définit l'intermédiaire subjectif (l'être tel que je le comprends) par lequel Autrui est relativisé à un autre moi, exige une hérésie, ou bien l'on montre qu'une autre phénoménologie sans horizon est possible - la phénoménologie de la donation de Marion et, peut-être, l'éthique de Levinas —, ou bien l'on affirme, comme Derrida, que "sans horizon, il n'y a pas de phénoménologie ", et l'on en tire toutes les conséquences - de ce point de vue, la déconstruction se comprend comme une alternative à la phénoménologie et au concept d'horizon qui la définit.

6. Sur le rapport de Levinas à l'esprit et à la lettre de la phénoménologie husserlienne, voir Derrida (I967 : I28-9n). 
Cependant, il ne s'agit là que du pan le plus tardif de l'histoire qui nous intéresse. Car, comme nous l'avons anticipé plus haut, le premier à avoir exigé le dépassement d'Être et Temps et l'abandon de l'horizon, ce n'est ni Derrida, ni Marion, ni même Levinas, mais Heidegger lui-même. Or, il faut chercher chez lui non seulement l'inauguration d'une définition de l'horizon que l'on retrouvera par la suite chez Levinas et dans le débat Derrida/Marion, mais également le modèle de "phénoménologie» que partage de façon souterraine l'ensemble des options philosophiques en jeu, pardelà leurs divergences apparentes (phénoménologie véritable sans horizon vs phénoménologie impossible sans horizon; phénoménologie de la donation de Marion vs déconstruction derridienne et éthique levinassienne). C'est ce que nous allons montrer à partir d'une analyse du dialogue fictif entre un savant, un professeur et un érudit: Pour servir de commentaire à Sérénité, écrit d'après les indications de Heidegger en I944-1945, puis publié en I959, soit deux ans avant Totalité et Infini.

Le dialogue commence par la formulation de la thèse selon laquelle «la question de l'essence de l'homme ", c'est-à-dire de la pensée, " [n'est] pas une question tournée vers l'homme» (GL:29/I49). Autrement dit, ce n'est pas en demandant à l'homme en quoi consiste son essence que l'on obtiendra quoi que ce soit à ce sujet, car d'une telle enquête ne ressortirait que le point de vue de l'homme sur cette dernière. C'est donc un certain modèle de la pensée, auquel Heidegger opposera plus loin la sérénité, qu'il critique, et qui est présenté sous les traits de l'herméneutique phénoménologique d'Être et Temps: ce qui est rejeté, c'est le mode de «la pensée sous la forme du représenter transcendantal-horizontal» (GL: trad. modifiée, 36/I 54), par lequel nous dépassons l'aspect des objets (= étants) vers un horizon (= l'être) qui constitue la perspective subjective délimitant la manière dont nous les comprenons, c'est-à-dire "seulement le côté tourné vers nous d'une ouverture qui nous environne» (GL: trad. modifiée, 37/I 55 ).

Mais il faut être ici encore plus précis. En effet, le caractère subjectif de l'horizon comme délimitation de la manière dont nous comprenons les objets est en vérité attribué rétrospectivement par Heidegger au modèle d'Être et Temps, c'est-à-dire à la lumière de sa critique, inconnue de l'œuvre de 1927 , de la métaphysique moderne de la subjectivité. L'Être et Temps qu'il s'agit de dépasser ici est une version révisée à l'aune des recherches contemporaines du second Heidegger, et dont le dépassement doit mener à une autre ontologie plus radicale, celle de l'être comme vérité, comme le confirme la remarque dans D'un entretien de la parole, selon laquelle ce qui est à penser, c'est " "l'être" relativement à son sens propre, c'est-à-dire à sa vérité (éclaircie) », par distinction avec "l'être de l'étant» (GS: IO4-I05).

Or c'est précisément ce geste du dépassement ou de la radicalisation qui est à l'œuvre dans ce dialogue : au contraire du représenter transcendantalhorizontal, la sérénité définit l'essence originaire de la pensée, qui consiste justement à penser non pas l'étant à partir de ce qui est pour nous (= l'être 
comme horizon = l'être de l'étant), mais l'ouverture «en soi [in sich]» (GL: 39/I 57), autre nom de la "contrée» ou de l'être comme vérité. En ce sens, le dépassement d'Être et Temps vers une figure plus originaire que l'être de l'étant implique corrélativement l'abandon de l'horizon, et explique de manière générale la disparition de la détermination du caractère phénoménologique et herméneutique de son entreprise philosophique après le tournant.

Ainsi se dégage de l'ensemble des analyses que nous venons de présenter une logique commune au Heidegger de Gelassenheit, à Levinas, Derrida et Marion, logique qui doit permettre de regrouper ces auteurs - la liste proposée n'est évidemment pas close - dans l'unité d'une histoire, et qui possède trois propriétés: La première, c'est que, par-delà la multiplicité des étiquettes — ontologie, éthique, phénoménologie, déconstruction —, et les différences dans le choix de l'objet philosophique qu'il s'agit de penser - l'être comme vérité, Autrui, la donation, la différance - , les auteurs considérés partagent une même conviction quant à ce que doit être la recherche philosophique. Or, par référence au $\mathbb{} 7$ d'Être et Temps dans lequel la définition explicite de cette manière de concevoir l'activité philosophique prend sa source, dans ce qui suit, nous appellerons ce type de philosophie «herméneutique phénoménologique»: faire de la philosophie, au sens le plus technique du terme, c'est laisser se montrer soi-même à partir de soi-même (logos = herméneutique) le phénomène par excellence (phénoménologie), c'est-à-dire ce qui de prime abord et le plus souvent ne se montre pas, mais est le fondement (= origine) de ce qui se montre de prime abord et le plus souvent (= dérivé) $(S Z: 35 / 47)$. Or, si dans Être et Temps une telle définition de la recherche philosophique coïncide avec l'explicitation du sens/horizon de l'être en général à partir d'une analytique du Dasein ( $S Z$ : $38 / 34$ ), on peut également la considérer formellement, c'est-à-dire en écartant le contenu matériel que lui assigne le $\mathbb{7} 7$, comme un thème susceptible d'une multiplicité de variations possibles, disposant à chaque fois d'un contenu distinct - le $\$ 7$ n'étant alors qu'une variante parmi d'autres de ce thème. Ainsi, ce qui unit autant Heidegger que Levinas, Derrida ou Marion, c'est qu'il s'agit pour eux de laisser se montrer le phénomène par excellence; et ce qui les différencie, c'est que le phénomène par excellence et la manière d'y accéder prennent à chaque fois des contenus différents: l'explicitation du sens de l'être, la sérénité à l'égard de l'être comme vérité, laisser s'ex-

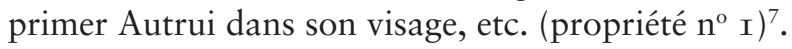

7. Pour une justification détaillée de l'usage de l'expression d'herméneutique phénoménologique et une analyse plus précise de ce qui la définit, la formule étant, on le voit, prise en un sens très précis qui, comme thème formel, ne coïncide pas avec telle ou telle entreprise philosophique se revendiquant explicitement de l'herméneutique phénoménologique (celle de Ricœur ou une autre), voir Djian/Majolino (20I8). Cette logique de la recherche du phénomène par excellence va d'ailleurs bien au-delà des cas que nous venons d'indiquer, et n'implique pas formellement le motif ultérieur, introduit par le second Heidegger, et par conséquent présent uniquement dans certaines variantes matériellement déterminées, du dépassement de 
À cette première propriété, on peut ajouter une seconde que ne partagent que certains herméneutes phénoménologues: la volonté de dépasser la phénoménologie d'Être et Temps revisitée à l'aune de la critique de la métaphysique moderne de la subjectivité, geste inauguré par Heidegger luimême, et que reprennent les phénoménologues français considérés (propriété $n^{\circ} 2$ ).

Corrélativement, le dépassement d'Être et Temps implique l'abandon de l'horizon, dont l'usage, repris par les phénoménologues ultérieurs, est défini par le second Heidegger, à la lumière de sa critique de la métaphysique de la subjectivité, comme perspective subjective délimitant pour nous ce que nous comprenons. Il s'agit dès lors d'opposer à la conception philosophique, subjectivisée après coup, d'Être et Temps une nouvelle tentative de laisser se montrer, non plus für uns mais in sich, une origine plus ultra (propriété $n^{\circ} 3$ ).

À partir de là, on peut déterminer la spécificité «française» — l'expression n'étant évidemment utilisée que par référence à Levinas, Derrida et Marion, qui sont les acteurs majeurs de ce pan de l'histoire de l'herméneutique phénoménologique, la liste des auteurs, et des nationalités, n'étant là encore pas close - de l'herméneutique phénoménologique, qui repose sur une propriété additionnelle: elle consiste dans le fait d'appliquer à l'ontologie heideggerienne comme telle ce que Heidegger exigeait seulement pour l'être de l'étant, à savoir l'argument du dépassement de l'horizon subjectif vers une origine plus ultra (propriété add. $\mathrm{n}^{\circ} 4$ ).

L'enquête généalogique que nous venons d'esquisser nous permet dès lors de tirer deux conséquences majeures pour notre travail.

D'abord, elle nous permet de comprendre pourquoi, dans un certain sens, le débat entre Derrida et Marion constitue un changement de perspective quant à la manière de concevoir l'histoire de ce qui avait été appelé jusqu'ici, dans un sens extrêmement indéterminé, "phénoménologie». Car si ce que nous venons de dire est juste, il apparaît en effet que l'histoire de l'horizon fait l'histoire de la phénoménologie: non seulement il renvoie à un type bien spécifique de phénoménologie, présenté dans le $\mathbb{S} 7$ d’Être et Temps et revisité après le tournant; mais, à partir du dépassement de cette version revisitée, l'abandon de l'horizon représente une fin de recevoir à la voie philosophique ouverte par Etre et Temps, et semble inaugurer une croisée des chemins qui préside au destin de la phénoménologie elle-même, soit à sa disparition, soit à sa vie véritable.

Mais, d'un autre côté, l'enquête généalogique a débouché sur l'exposition d'une logique commune aux phénoménologues français et à Heidegger

l'horizon et de la métaphysique moderne de la subjectivité. C'est pourquoi ce que nous appellerons plus loin le pan "français" de l'histoire de l'herméneutique phénoménologique, qui implique un tel dépassement, possède une situation spécifique dans l'histoire générale que nous allons chercher à déterminer. 
(celui d'Être et Temps comme celui de Gelassenheit), l'herméneutique phénoménologique, qui, en dépit de la spécificité du versant "français ", exige de réviser, non pas la thèse de l'entrelacement de l'histoire de la phénoménologie et de l'horizon, mais la perspective esquissée à son propos dans le débat Derrida/Marion. Car, dès lors que l'on peut regrouper formellement ces auteurs par référence au thème de l'herméneutique phénoménologique, les prétentions respectives à la radicalité et au dépassement, les différences de bannières sous lesquelles ceux-ci se présentent et a fortiori le débat sur la possibilité, ou non, d'une phénoménologie sans horizon, qui ne serait rien d'autre que la phénoménologie véritable - tout cela apparaît, dans un autre sens, comme un épiphénomène qui masque la persistance souterraine du même thème herméneutico-phénoménologique derrière la multiplication des variantes. De ce point de vue, par-delà la différence apparente résultant des décisions respectives prises à l'égard de la pseudo-croisée des chemins dont nous parlions plus haut, la démarche unitaire de Levinas, Derrida et Marion se caractérise par l'accélération d'un pan de l'histoire de l'herméneutique phénoménologique que le geste de dépassement d'Être et Temps, formulé par Heidegger lui-même, avait d'abord amené à un premier point culminant: il s'agit d'exiger, à travers le dépassement de la version revisitée d'Être et Temps, celui de l'ontologie heideggerienne comme telle, vers une figure plus originaire (Autrui, la différance, la donation, etc.). On appellera donc «histoire française de l'herméneutique phénoménologique» le pan spécifique de l'histoire de l'herméneutique phénoménologique, magistralement inauguré dans Totalité et Infini par Levinas, qui implique l'exigence de dépassement que nous venons de définir. Dès lors, on peut se servir du motif du dépassement de l'horizon - pour autant que le concept est considéré comme propre à l'ontologie heideggerienne comme telle, et que le motif manifeste la tentative de dépasser Heidegger en général - comme d'un critère sûr permettant de faire voir une telle accélération, et de décider de l'appartenance ou non de telle ou telle doctrine au pan dit «français" de l'herméneutique phénoménologique. On arrive ainsi à la seconde hypothèse de ce travail, à savoir que l'histoire de l'horizon doit pouvoir servir de fil directeur pour reconstruire le sens de ce versant français.

Le principe d'une telle reconstruction étant posé, il reste à commencer à l'appliquer, ce qui permettra à la fois de le justifier et d'esquisser les résultats que l'on pourrait en tirer. L'enjeu étant limité et n'exigeant évidemment pas l'exhaustivité, nous n'aborderons ici que deux cas. Celui de Levinas ayant déjà été en grande partie examiné ${ }^{8}$, et celui de Derrida pouvant l'être aisément à partir de l'article de Eddo Evink, «Horizons of Expectations.

8. Pour une confirmation du caractère herméneutico-phénoménologique - l'expression étant prise au sens technique défini plus haut - de l'entreprise philosophique de Totalité et Infini, et quelques autres exemples de philosophies de ce type (Gadamer, Ricœur et Marion, sur lequel nous reviendrons plus loin), voir Djian et Majolino (20I 8). 
Ricœur, Derrida, Patocka ", qui propose déjà une analyse générale des usages derridiens de l'horizon, nous étudierons deux textes: L'essence de la manifestation de Michel Henry et Étant donné de Jean-Luc Marion, dans lesquels on retrouve explicitement l'exigence d'un dépassement de l'horizon, et dont l'analyse successive doit permettre d'esquisser la direction vers laquelle l'histoire "française» semble tendre: celle d'une accélération au second degré, c'est-à-dire d'une recherche tout à fait originale visant à laisser se montrer le phénomène par excellence des phénomènes par excellence en l'occurrence, la donation.

\section{L'histoire française de la phénoménologie}

\section{a) L'essence de la manifestation de Michel Henry}

De façon analogue à Totalité et Infini, l'entreprise philosophique de Henry se veut une confrontation avec les présuppositions du «monisme ontologique » dont les "présuppositions commandent, depuis son origine en Grèce, le développement de la pensée philosophique occidentale»(EM:9I). La thèse principale du monisme, que Henry formule dans le vocabulaire d'Etre et Temps, consiste en effet à affirmer que l'essence de la manifestation l'origine comme "ce à partir de quoi un pouvoir [quel qu'il soit] est possible, ce à partir de quoi il se développe, de telle manière cependant qu'il ne peut jamais revenir sur elle, jamais la reprendre» (EM: 368$)$ - n'est et ne peut être que la transcendance, qui définit le mode de déploiement du " "phénomène" comme quelque chose qui se montre dans l'horizon de lumière à l'intérieur duquel toute chose peut devenir visible en elle-même " (EM: $5 \mathrm{I}$ ), qu'il s'agisse de l'horizon de l'être (qui «phénoménalise» l'étant, voir EM: 29) ou de l'horizon de la temporalité propre à la transcendance du Dasein (qui "phénoménalise" l'être, voir $E M: \mathbb{S} 23-24$ ). Or, aux yeux de Henry, la détermination du phénomène comme ce qui se montre intuitivement (EM: 37 ) dans un horizon (de l'être ou du temps) est « unilatérale» et doit être complétée par une autre conception du phénomène, dont «le mode même conformément auquel il se révèle est irréductible au "comment" de la manifestation des phénomènes transcendants" (EM: 5I), et qui doit ouvrir la voie à une autre phénoménologie, plus fondamentale que celle de la transcendance (cf. le titre du $\mathbb{S I I}$, "Le monisme ontologique et le problème de son dépassement ", qui conduit à la section II, «Répétition de l'élucidation du concept de phénomène. Transcendance et immanence»). Cet autre «comment », qui n'est pas l'horizon ou le sens objectivé par le Dasein dans sa transcendance, et qui conduit à un phénomène non transcendant (= l'ego), c'est l'immanence ou la "révélation » (EM: 52): s'il est vrai, en effet, que l'être en général se manifeste dans la transcendance du Dasein, il y a une condition plus fondamentale que la transcendance dans laquelle celle-ci se manifeste; cette essence originaire de la manifestation de la transcendance ne peut pas être la transcendance elle-même $(E M: 258 ; 279)$, sans quoi on tomberait dans un cercle, mais un mode de manifestation plus originaire, 
renvoyant à une autre conception du phénomène - et à une autre phénoménologie (EM: 54). Ainsi, la phénoménologie de la transcendance repose sur un concept de phénomène définissant ce qui se manifeste intuitivement dans l'horizon objectivé et distancé (voir l'analyse du concept de «distance phénoménologique» aux $\mathbb{S}$ 9-Io) de la transcendance du Dasein; au contraire, la phénoménologie de l'immanence ou de la vie (EM: 568) est fondée sur un autre concept de phénomène, caractérisé par son autorévélation sans distance, sans distinction par rapport à un horizon dans lequel il se montrerait, c'est-à-dire par le contact immédiat de l'ego avec soi-même (EM: 279-80), contact non intuitif, sur le mode de " "l'invisible" " comme «mode d'une révélation positive et, à vrai dire, fondamentale» (EM: 57).

On a ainsi retrouvé trois des quatre propriétés qui caractérisent le pan «français» de l'histoire de l'herméneutique phénoménologique: Henry exige le dépassement du monisme ontologique, présenté sur le modèle métaphysique de la version revisitée d'Être et Temps - le monisme, qui a dominé l'ensemble de la tradition philosophique occidentale, réduit tout mode de manifestation à la transcendance, où le phénomène apparaît intuitivement dans l'horizon de l'être que l'ego s'est opposé - en vue d'une essence de la manifestation plus originaire, qui conditionne la manifestation de la transcendance elle-même, à savoir l'immanence (propriété $\mathrm{n}^{\circ} 2$ ). Ce dépassement qui, conformément à la caractéristique propre du volet «français » de l'herméneutique phénoménologique, vise l'ontologie heideggerienne comme telle (propriété add. $\mathrm{n}^{\circ} 4$ ), exige une autre phénoménologie, fondée sur un autre concept de phénomène, qui ne se manifeste plus de manière intuitive dans l'horizon subjectif projeté dans la transcendance du Dasein, mais se révèle à lui-même sans distance. Le dépassement de la version revisitée d'Être et Temps coïncide donc avec l'abandon de l'horizon, horizon qui caractérise le mode de manifestation de la transcendance (propriété $\mathrm{n}^{\circ} 3$ ). Il reste dès lors à démontrer la propriété $\mathrm{n}^{\circ} \mathrm{I}$, à savoir que la phénoménologie de la vie présentée dans L'essence de la manifestation correspond à la définition formelle que nous avons donnée de l'herméneutique phénoménologique, comme laisser se montrer le phénomène par excellence, c'est-à-dire ce qui de prime abord et le plus souvent ne se montre pas, mais est le fondement de ce qui se montre.

Commençons par le phénomène par excellence. D'abord, s'il est vrai que la vie de l'ego se révèle immédiatement sans différence ou distance, «ce qu'il y a de plus simple et de plus "évident", nous savons depuis longtemps que c'est aussi ce qu'il y a de plus "difficile" " $(E M: 55)$. Et cette difficulté à étudier ce qu'il y a de plus simple est fondée sur le retrait qui est propre à cette vie: la dissimulation de l'essence originaire de la révélation, ce «qui fait, dans la structure interne de l'immanence, qu'elle se dérobe » $(E M: 479)$, c'est que l'être de l'ego, étant la condition de la manifestation de toute transcendance, c'est-à-dire du monde et des étants intramondains, mais étant 
dans le même temps hétérogène à toute transcendance, ne s'y manifeste pas lui-même ( $E M: 480 / 380$ ). Si l'on ajoute à ce premier point l'oubli structurel de la vie, fondé, comme chez Heidegger, sur la déchéance du Dasein qui le voue à la "tentation de l'étant» $(E M: 25 ; 483-4)$, on retrouve l'ensemble des traits caractérisant le phénomène par excellence: la vie est le fondement de ce qui se montre, comme fondement (originaire) de soi-même et (dérivé) du monde, qu'il manifeste en se retirant structurellement dans la Nuit comme aime parfois à le dire Henry (EM: 554/44I-2) —, c'est-à-dire dans l'invisible; elle est certes originairement révélation de soi-même, mais, de prime abord et le plus souvent en raison de la tendance structurelle de la pensée à se vouer à l'étant et à comprendre la vie à partir de lui, elle est cachée. C'est pourquoi cette vie, comme phénomène par excellence, exige une phénoménologie, plus précisément un logos qui le laisse se montrer soimême à partir de soi-même; la vie étant un phénomène immanent, autant l'intuition que la compréhension quotidienne sont exclues, et il ne reste qu'à tenter de penser dans un «Re-mémorial authentique » le retour, par-delà les falsifications structurelles, à "ce qu'elle dit spontanément d'elle-même dans la vérité du langage naturel» (EM: 489), à ce qu'elle a toujours dit mais qui a toujours déjà été quotidiennement falsifié (EM: 688; 69I).

Après Totalité et Infini, l'entreprise philosophique de L'essence de la manifestation constitue donc bien une seconde variante de l'histoire «française " de l'herméneutique phénoménologique: elle définit une herméneutique phénoménologique ayant pour but de laisser se montrer la vie comme phénomène par excellence (propriété $\mathrm{n}^{\circ} \mathrm{I}$ ), qui implique le dépassement du monisme ontologique présenté sous les traits de la version revisitée d'Être et Temps (propriété $\mathrm{n}^{\circ} 2$ ), c'est-à-dire de l'ontologie heideggerienne comme telle (propriété add. $\mathrm{n}^{\circ} 4$ ); tentative de dépassement qui est rendue apparente par l'exigence d'abandonner l'horizon pour autant qu'il qualifie le mode de manifestation de la transcendance (propriété $\mathrm{n}^{\circ} 3$ ), et qui doit déboucher sur un fondement plus originaire que l'être comme horizon, à savoir l'auto-manifestation de la vie elle-même.

\section{b) Étant donné de Jean-Luc Marion}

Si la présence du motif de l'abandon de l'horizon en vue d'une figure plus originaire que l'être heideggerien a permis de rendre visible l'appartenance de L'essence de la manifestation à l'histoire française de l'herméneutique phénoménologique, on y trouve aucune tentative de déterminer les rapports éventuels que la vie entretient avec les autres variantes de phénomène par excellence proposés par les acteurs herméneutes phénoménologues contemporains de la parution du livre, à savoir l'être comme vérité heideggerien ou Autrui, qui sont complètement ignorés - bien que l'un comme l'autre ne puissent être assimilés, dans le langage d'Henry, à un phénomène transcendant, qu'il s'agisse de l'étant, c'est-à-dire du monde, ou de l'être de l'étant comme horizon. De ce point de vue, Étant donné de Marion, qui va nous 
intéresser à présent, possède une place très singulière dans l'accélération dite «française» de l'herméneutique phénoménologique. Il s'agit en effet de la première tentative de rediriger une nouvelle fois le motif heideggerien du dépassement, cette fois non plus exclusivement contre l'ontologie heideggerienne comme telle, mais à l'égard des variantes de phénomène par excellence supposées avoir dépassé cette dernière. Autrement dit, Marion ne cherche pas simplement à introduire une nouvelle variante du phénomène par excellence; il veut articuler l'ensemble des figures originaires qui ont précédé Étant donné, c'est-à-dire dégager entre elles une unité de type $\pi \rho \varsigma$ Èv, par référence à un phénomène par excellence plus originaire: la donation. Ainsi, comme le souligne Marion dans les dernières lignes de la conclusion: "loin de méconnaître ici les avancées les plus récentes de la phénoménologie - auto-affection (Henry), herméneutique (Ricœur), différance (Derrida) et regard d'autrui (Levinas) - , nous tentons simplement de les confirmer en assignant à chacune son site précis dans la donation " (ED: $52 \mathrm{I})$. La tentative d'articuler l'ensemble des variantes précédentes du phénomène par excellence à un phénomène par excellence plus ultra définit donc la place particulière qu'occupe Marion dans l'histoire française de l'herméneutique phénoménologique, et le geste du dépassement vers une figure plus originaire, sa place tout court dans cette histoire. Conformément à notre méthode, c'est le motif de l'abandon de l'horizon qui doit rendre ce dernier geste apparent, c'est donc lui qui nous intéresse ici. Mais avant cela, pour clarifier la théorie générale de la donation, nous commencerons par vérifier l'appartenance de l'entreprise d'Étant donné au thème de l'herméneutique phénoménologique (= propriété $\left.\mathrm{n}^{\circ} \mathrm{I}\right)$.

Le point de départ d'Étant donné est l'opposition, formulée au $\mathbb{S} 7 \mathrm{c}$ d'Être et Temps, entre phénomène et être-recouvert. Le premier définit le mode de "ce qui se montre à partir de soi-même comme pure apparition de soi sans reste» $(E D: 303)$, "automanifestation » $(E D: 359)$, supposé définir la donation comme fondement du donné, ou donner du don (ED:6I), c'està-dire du phénomène: il y a une «équivalence foncière [de la donation] avec la phénoménalité - ce qui apparaît se donne, ce qui se donne apparaît, ou mieux se montre» (ED: I97). Le second, que Marion appelle «apparence», désigne une dégradation du phénomène dans le cadre de la "représentation objectivante" $(E D: 22 ; 83 ; 85)$ solidaire du "privilège de la perception et de la subjectivité (métaphysiques)» (ED: I3). En effet, alors que le phénomène se montre en soi, l'apparence est le phénomène tel qu'il se montre pour nous, tel que «la subjectivité [l']aperçoit par tel ou tel de ses outils perceptifs", qui ainsi «filtre, interprète et déforme l'apparence de l'apparition" (ED: I2). Le rapport avec Etre et Temps est cependant doublement compliqué, d'abord par l'identification entre phénomène et phénomène par excellence - seul le "phénomène par excellence» (ED:3II) se montre, c'est-à-dire les phénomènes saturés (l'événement, l'idole, la chair, l'icône, la révélation), qui sont définis par l'excès d'intuition sur l'intention $(E D: 325)$, 
tandis que les phénomènes pauvres (essences) et communs (objets de la physique et des sciences de la nature, objets techniques) ne sont qu'apparences -, ce qui n'empêche pas Marion d'appeler «phénomène» de telles apparences; et ensuite parce que la donation du phénomène est, dans "l'attitude naturelle» (ED: I 88-9; 290), ou dans l'attitude de «sens commun» (ED: I42) de prime abord et le plus souvent cachée. Or, la première difficulté est justement réglée par le caractère de retrait du phénomène: de prime abord et le plus souvent, la donation du phénomène est masquée et limitée à une "possibilité bornée de la phénoménalité ", celle de l' "objectivité finie " (ED: 324); les phénomènes pauvres ou communs sont certes des phénomènes, mais des phénomènes "handicapés" ou "offusqués" (ED:520), montrés subjectivement dans une "phénoménalité aliénée » $(E D: 302)$. Mais si l'attitude naturelle est première temporellement, ce qui explique "le privilège, presque toujours accordé par les théories de la connaissance (de Platon à Descartes, de Kant à Husserl) " aux phénomènes pauvres et communs (ED: $32 \mathrm{I}$ ), originairement en revanche la donation du phénomène est libre, c'està-dire que l'intuition y est en excès par rapport à l'intention, de telle manière qu'il faille «renverser la définition commune du phénomène » $(E D: 324)$ en prenant comme paradigme le phénomène qui est à la fois le moins commun, le plus exceptionnel, mais également celui qui montre le mieux à quoi ressemble le phénomène comme tel, à savoir le phénomène saturé.

La donation réunit ainsi tous les traits de ce qu'on a appelé phénomène par excellence: il est le fondement, constamment en retrait, de ce qui se montre, c'est-à-dire du phénomène, qui est d'abord dissimulé par la limitation de la phénoménalité à celle de l'étant ou de l'objet, masquant l'universalité de la donation; c'est pourquoi il exige de se laisser montrer dans un logos dont Marion concède qu'il est herméneutique: "reconnaître ainsi le don [= tel qu'il se donne] implique un regard phénoménologique, strict et particulier: celui qui, devant le fait, le voit comme un don. Il s'agit, si l'on veut, d'herméneutique, mais d'une herméneutique qui donne moins un sens qu'elle ne le reçoit pour précisément entendre un don. Moins don du sens que sens du don» (ED: 187$)$.

Ce qui fait alors l'originalité d'Étant donné, comme nous l'avons anticipé plus haut, c'est que la donation ne s'ajoute pas seulement comme une énième variante à celles de Levinas et de Henry. Au contraire, elle désigne le phénomène par excellence fondant à la fois les phénomènes vulgaires et l'auto-manifestation des phénomènes saturés, c'est-à-dire des phénomènes que Marion appelle lui-même "par excellence» (ED:3 II), qui renvoient tous (hormis le cas de la révélation) à une "phénoménologie» déjà accomplie - la phénoménologie de l'événement de Ricœur, celle de l'idole de Derrida, celle de la chair de Henry (et sans doute du second Merleau-Ponty), enfin celle de la phénoménologie de l'icône de Levinas - , les unes se distinguant des autres par la détermination du phénomène saturé sur laquelle ils insistent (respectivement quantité, qualité, relation, modalité, voir ED: 
\2I-22). Par là, non seulement les phénomènes pauvres, communs et saturés peuvent être organisés à partir de leur référence $\pi \rho$ ò $̧$ ह̂v à la donation, comme donner le don qui se retire $(E D: 60)$; mais la donation constitue en vérité le phénomène par excellence plus ultra par rapport auquel les précédentes tentatives phénoménologiques de cerner l'originaire sont à la fois dépassées et rendues intelligibles dans leur architecture générale ( $E D: 52 \mathrm{I})$. En ce sens, la donation se veut la variante des variantes du phénomène par excellence - et la phénoménologie de la donation la variante des variantes d'herméneutiques phénoménologiques.

Nous avons à présent les moyens de déterminer le rôle de l'horizon dans l'économie générale du livre. Celui-ci définit en effet, comme nous allons le voir en détail, la condition de possibilité de la phénoménalité du phénomène, la finitude de l'intuition, et participe, comme délimitation du non vu en prévisible, du privilège de la vision et de la représentation que la phénoménologie doit dépasser (propriété $\mathrm{n}^{\circ} 3$ ). Il suffit dès lors de montrer que, en dépit de la référence constante que fait Marion à la théorie husserlienne de l'horizon, c'est bien de la critique heideggerienne de l'horizon comme limite subjective délimitatrice dont il est question (propriété $\mathrm{n}^{\circ} 2$ ), et avec l'abandon de l'horizon du dépassement de l'ontologie heideggerienne comme telle (propriété add. $\mathrm{n}^{\circ} 4$ ), pour garantir la place d'Étant donné dans le versant français de l'histoire de l'herméneutique phénoménologique.

$\mathrm{Si}$, aux yeux de Marion, la théorie husserlienne du principe des principes constitue une étape importante dans la libération de la phénoménalité de son aliénation (ED:302-303), la tentative de Husserl échoue pourtant en raison du maintien de la thèse d'une "finitude de l'intuition elle-même", finitude qui est à la fois de fait et de droit: de fait car, dans la phénoménologie husserlienne, l'évidence adéquate où se recouvrent complètement l'intention et l'intuition reste «la plupart du temps [...] un cas limite, voire exclu» (ED: 3I4); de droit puisque l'intuition est encadrée par "deux conditions de possibilité » qui "sapent la prétention à la possibilité absolue ouverte par l'intuition donatrice» (ED: 304), à savoir l'horizon et le Je. De ce point de vue, quelle que soit pour l'instant la signification de ce concept, si l'horizon aliène la donation, la réévaluation de celle-ci implique un mode de manifestation irréductible à celui de l'apparence dans l'horizon.

En quoi la critique que Marion fait de l'horizon reprend-elle alors le cadre général de la critique heideggerienne de l'horizon subjectiviste, solidaire de l'analyse de la métaphysique moderne de la subjectivité ? Pour le voir, il faut détailler la définition husserlienne de l'horizon, telle qu'elle est formulée par Marion : «toute intuition, pour donner dans certaines "bornes" de fait, doit s'inscrire d'abord de droit dans les "limites" [Grenze] d'un horizon, de même qu'aucune visée intentionnelle d'objet, de signification ou d'essence ne peut s'exercer hors d'un horizon" (ED: 305). Le trait de la limite, devenu après le tournant la caractéristique principale de l'horizon subjectiviste attribué rétrospectivement par Heidegger à l'herméneutique 
phénoménologique d'Être et Temps, nous met ici sur la voie pour fixer l'arrière-plan de la discussion, dont il est clair qu'il n'est pas strictement husserlien'. Le point de départ husserlien est donc moins une nécessité qu'une occasion pour retrouver chez ce dernier, malgré la discussion du principe des principes, l'horizon subjectiviste propre à la métaphysique moderne, qui permet de justifier l'exigence de son dépassement, et avec lui le dépassement de l'ontologie heideggerienne comme telle (et de la phénoménologie husserlienne, que Heidegger avait préalablement radicalisée [ED: 46-67]), dans le but de libérer le phénomène par excellence de son carcan. Dès lors, le caractère de limite de l'horizon est triple.

I. D'abord, s'il est vrai que Husserl a confondu «la donation elle-même avec l'objectité » (ED:54), tout ce qui est donné est pensé par ce dernier comme un "objet», dont le modèle est la chose constituée par un Je $(E D: 55 ; 305)$, de telle manière que sa phénoménalité est limitée à l'intuition sensible: l'objet est ma maison que je vois de ce côté, la scène de spectacle que je me souviens avoir vue de la fosse où j'étais placé, la plage italienne que je m'imagine parcourir, etc. De ce point de vue, l'évidence correspondant à la perception de la chose est par principe inadéquate, c'est-à-dire implique qu'il reste toujours — c'est là un aspect de la définition proprement husserlienne de l'horizon perceptif - un horizon de vécus de visée non remplis qui déborde la perception actuelle de l'une ou l'autre des faces de la chose. Autrement dit, dans l'inadéquation perceptive est visé plus que ce qui est donné. Or c'est précisément cette raréfaction ou limitation de l'intuition/donation sur la visée (ou l'intention vide) qui permet, par une opération philosophique propre à la théorie de Marion, mais inconnue de la description husserlienne, de parler de finitude de droit de l'intuition chez Husserl: ce qui était, aux yeux de ce dernier, une structure eidétique de la corrélation perceptive comme telle, qu'il s'agisse de notre perception, de celle de Dieu ou de n'importe quelle conscience non finie que l'on peut s'imaginer pour autant qu'elle perçoit (= la perception implique l'horizon comme structure rendant possible, en débordant sur les vécus inactuels, passés et à venir, la synthèse de la perception) devient avec Marion une limite, c'està-dire l'expression de notre finitude: dans la perception de la chose «l'intuition reste essentiellement défaillante, pauvre, nécessiteuse, indigente $-\pi \varepsilon v i ́ \alpha$. L'adéquation entre l'intention et l'intuition devient donc une simple limite, un idéal habituellement évoqué par défaut» (ED: 3I5). Ainsi, la réduction de la phénoménalité à celle de l'objet, qui manifeste le primat de l'intention sur l'intuition, masque la donation, qui consiste au contraire dans la saturation de l'intuition supposant, comme nous allons le voir, la saturation de l'horizon (ED: 344-348).

9. La justification complète d'une telle thèse implique évidemment une analyse détaillée de l'usage husserlien d'horizon, qui fera l'objet d'un travail séparé. 
2. Cela étant, il ne s'agit là que d'un premier aspect du caractère de finitude de l'horizon. La détermination de l'horizon comme limite n'exprime pas seulement la finitude de droit de l'intuition par rapport à l'intention dans notre perception; s'appuyant sur l'étymologie grecque du mot, Marion affirme qu'il désigne également la «délimitation» de l'intuition à venir, de l'inconnu inactuel au connu, de la «nouveauté irrépressible du flux des vécus, donc de l'intuition» (ED: 305-6) au « rang d'un pré-vu, d'un visible simplement retardé, sans nouveauté foncièrement irréductible, bref d'un pré-visible» (ED: 307). Ainsi, contrairement à l'événementialité imprévue de la donation, l'intuition de la chose «reste, en droit, toujours comprise dans un horizon déjà défini ", qui délimite, c'est-à-dire aliène, les intuitions à venir pour qu'elles répondent aux conditions de l'expérience de la chose (ED: 306). L'intuition est donc finie dans un double sens: d'abord, parce qu'elle est structurellement dépassée par l'intention sous la forme de l'horizon; ensuite, parce que l'horizon réduit ou limite le non encore donné, la donation future, de l'objet à un pré-visible sans imprévu ni accroc, qui masque l'événement de la donation. Cette double finitude est d'ailleurs corrélée à la seconde condition imposée par Husserl à la phénoménalité dans le principe des principes, à savoir le Je transcendantal qui exige que " toute intuition ne présente ce qui apparaît qu'en le donnant “... à nous" ", de telle manière que «la donation du phénomène à partir de soi à un $J e$ peut à chaque instant $[. .$.$] virer vers une$ constitution du phénomène par et à partir du Je» (ED: 308).

On retrouve ainsi l'opposition, que nous avons vue à l'œuvre dans Pour servir de commentaire à Sérénité, entre le modèle de la métaphysique moderne de la subjectivité, dont le projet de domination de l'étant exige la relativisation de l'horizon de l'être tel qu'il est compris pour nous - modèle qui, selon Marion, privilégie un paradigme du phénomène (le phénomène pauvre ou commun) qui a dominé l'histoire de la philosophie depuis Platon (ED: $32 \mathrm{I})$ - et la nécessité de le montrer en soi, c'est-à-dire soi-même à partir de soi-même (ED: I 3-4) (propriété $\mathrm{n}^{\circ} 2$ ). Ici, l'objet est le résultat d'une telle réduction de la donation pour nous par l'intermédiaire de la double condition de l'horizon subjectiviste et du Je auquel il se rapporte; et la phénoménologie de la donation a, semble-t-il, précisément pour but de dépasser le mode de la manifestation dans l'horizon (= propriété $\mathrm{n}^{\circ} 3$ ), et avec lui l'ontologie heideggerienne comme telle (= propriété add. $\left.\mathrm{n}^{\circ} 4\right)$ et l'ensemble de la philosophie occidentale, et de libérer la phénoménalité aliénée pour découvrir enfin le fondement originaire de ce qui se montre.

3. La place de l'entreprise philosophique d'Étant donné dans le pan français de l'histoire de l'herméneutique phénoménologique, et la situation particulière qu'elle occupe dans celle-ci, semblent à présent fixées. Mais 
l'examen du concept d'horizon, lui, n'est pas terminé, et la détermination du troisième sens de limite de l'horizon va exiger de revoir la manière que nous avons eue de considérer la situation de ce livre dans l'histoire en question. Jusqu'ici, en effet, la position soutenue dans ce texte semble correspondre à la thèse formulée par Marion dans son débat avec Derrida, à savoir que l'abandon de l'horizon est la dernière étape en vue d'une phénoménologie véritable - la phénoménologie de la donation. Cependant, en dépit du fait que le phénomène par excellence ne se montre que pour autant que l'intuition dépasse l'intention et l'horizon, Marion remarque qu'on ne saurait malgré tout «se dispenser d'un horizon en général, puisqu'on s'interdirait sans doute ainsi toute manifestation; il s'agit d'en user sur un autre mode, afin de se libérer de son antériorité délimitatrice, telle qu'elle ne peut qu'entrer en conflit avec la prétention d'un phénomène à l'apparaître absolu $(E D: 344)$. Cette thèse, qui semble relativiser l'affirmation "téméraire» de Marion lors du débat, doit donc être examinée pour finir cette analyse, ce qui implique de préciser le concept de saturation que nous avons laissé sous-déterminé jusqu'ici.

La saturation désigne aux yeux de Marion un cas de donation que la définition commune du phénomène chez Husserl suggère mais ignore, à savoir le surcroit d'intuition sur l'intention (ED: 325$)$. Or cette saturation est seule à même de rendre compte d'une donation et d'une automanifestation du phénomène: si l'horizon, comme instance $\mathrm{d}^{\mathrm{d}}$ " antériorité délimitatrice» $(E D: 344)$ corrélée au Je et réduisant le non vu à un prévisible, aliène le phénomène en tant qu'objet pour nous, la saturation d'intuition du phénomène implique au contraire l'impossibilité d'une telle antériorité, qui n'est possible que sur la base d'un surplus d'intention horizontale sur l'intuition. Trois cas de saturation peuvent alors être distingués: d'abord, celle où «le concept ou la signification de l'objet coïncident exactement avec les limites de son horizon, sans réserve d'espace signitif non encore rempli; l'intuition atteint non seulement l'adéquation avec la signification, mais la comble ainsi que tout son horizon» $(E D: 344)(=$ la chair de Henry et du second Merleau-Ponty); le deuxième cas, "au contraire du cas précédent", outrepasse "toute délimitation d'horizon. Cette disposition n'implique pas de se dispenser sans plus d'horizon, mais au contraire d'en articuler plusieurs ensemble pour accueillir un même et unique phénomène saturé » (ED: 345$)$ (= l'événement historique de Ricoeur, l'idole derridien, l'icône levinassien); enfin, il y a le cas de la révélation, "rare mais inévitable", où "la saturation redouble en bloquant les deux premiers cas sur elle ", c'est-à-dire où "les éblouissements dans chaque horizon" s'additionnent plutôt qu'ils ne se combinent $(E D: 347)$.

Ainsi, chaque figure de la saturation réside dans le fait, non pas d'être une intuition sans horizon, mais de saturer un, plusieurs, voire d'additionner les saturations d'une infinité d'horizons. Dès lors, pourquoi une telle satura- 
tion d'horizon, plutôt qu'un abandon de celui-ci? Dans quelle mesure s'interdirait-on toute manifestation s'il n'y avait pas d'horizon, et faut-il «en user sur un autre mode, afin de se libérer de son antériorité délimitatrice» (ED : 344), c'est-à-dire sur le mode de la saturation? C'est ici qu'intervient le troisième et dernier pan de l'usage de l' «horizon-finitude» chez Marion.

En effet, la manifestation du phénomène ne se produit que lorsque l'adonné, c'est-à-dire la nouvelle figure de la subjectivité corrélative de l'élévation de la donation au rang de principe du phénomène, reçoit le phénomène donné: «ce qui se donne ne se montre qu'autant qu'il se trouve reçu par l'adonné, dont la fonction propre consiste à donner en retour au donné de se montrer» (ED: 503). Or, même l'excès d'intuition «se trouve toujours mis en œuvre dans la finitude essentielle de l'adonné » (ED: 502), de telle manière que "pareille conversion du donné en phénomène montré ne peut [...] se réaliser que dans le champ, évidemment fini, où l'adonné reçoit et met en scène" (ED: 503-504). Ainsi, le phénomène donné peut "parfois, voire souvent, ne pas se régler sur la capacité de réception de l'adonné et en franchir les limites » (ED: 502). Autrement dit, si, pour nous, toute manifestation n'est possible que dans les limites de notre capacité de réception, cela signifie que l'on ne saurait faire l'impasse sur un horizon, c'est-à-dire précisément sur notre capacité limitée de réception dans laquelle la manifestation du phénomène est possible - que l'intuition soit en pénurie ou en excès sur lui.

On peut alors conclure: «si tout ce qui se montre doit d'abord se donner, il advient parfois que ce qui se donne ne parvienne pourtant pas à se montrer» $(E D: 502)$. Et, s'il est vrai que l'historicité de l'adonné implique qu'il ne saurait y avoir de répons, c'est-à-dire de manifestation du donné de la part de l'adonné, en dehors des limites imposées par l'horizon, il y a des raisons de croire que ce qui se donne ne se montre jamais comme tel, ce qui implique que "ces limites puissent reculer, voire reculer sans cesse ni fin" (ED: 503). Ainsi s'impose l'idée d'une inadéquation radicale de la manifestation, le phénomène donné reçu par l'adonné ne montrant «ce qu'il donne qu'inadéquatement, au long d'une série sans fin de répons toujours partiels et provisoires, manquant la défaillance irrévocable de l'adonné [...]. L'excès de la donation implique évidemment le retard sans fin du répons " (ED: 498). Autrement dit, le répons de l'adonné au don qu'il reçoit, par lequel il le met en scène, est infiniment inadéquat. C'est pourquoi Marion souligne que le recul des limites n'est pas une progression, "n'invalide pas cette finitude essentielle, mais au contraire la confirme» (ED: 503). En ce sens, c'est bien à une relativisation de la thèse formulée dans le débat avec Derrida qu'on assiste ici: l'horizon subjectiviste que l'on croyait avoir dépassé dans la saturation par laquelle le phénomène se montre et se donne est, à la fin des fins, assumé, pour satisfaire à la finitude qui est la nôtre. C'est là toute l'ambiguité de la situation d'Étant donné dans l'histoire "française" de l'herméneutique phénoménologique, que la méthode d'analyse que nous 
avons choisie, fondée sur l'usage du thème formel de l'herméneutique phénoménologique et de l'histoire de l'horizon comme fil directeur, a permis de suggérer.

\section{Conclusion}

Les analyses que nous venons de proposer ont permis, en prenant l'usage du concept d'horizon comme fil directeur heuristique, d'esquisser le sens d'un pan de l'histoire générale de l'herméneutique phénoménologique, celui de son accélération "française» qui, avec la phénoménologie de Marion, semble prendre une nouvelle direction, celle d'une radicalisation au second degré de cette accélération. De ce point de vue, il est certain que le pan «français» auquel nous nous sommes limité, et l'histoire générale ellemême, restent encore à écrire dans le détail; mais nous disposons à présent de principes pour le reconstruire, dont nous avons pu commencer à démontrer la valeur heuristique dans ce travail, à savoir l'usage du thème formel de l'herméneutique phénoménologique et l'utilisation de l'histoire de l'horizon comme fil directeur. Mais si l'hypothèse que nous avons suivie est juste, on peut également en tirer un fil différent mais complémentaire de réflexion historique et philosophique qui va nous permettre d'élargir la perspective de ce travail au-delà de l'histoire d'un certain type de phénoménologie, l'herméneutique phénoménologique.

En effet, le regroupement de certains auteurs par référence au thème formel de l'herméneutique phénoménologique, qui définit l'entreprise philosophique comme la tentative de laisser se montrer soi-même à partir de soimême le phénomène par excellence, et qui a permis de dévoiler le caractère d'épiphénomène du débat entre Derrida et Marion sur le soi-disant destin croisé de la phénoménologie et de l'horizon - «il y a une phénoménologie sans horizon, c'est la phénoménologie véritable» vs «il n'y a pas de phénoménologie sans horizon, il faut donc l'abandonner» - , doit permettre de poser franchement la question: le pouvoir d'intégration de ce modèle heuristique de l'herméneutique phénoménologique est-il suffisamment important pour subsumer toutes les phénoménologies qui ont émergé depuis plus de deux siècles déjà, de la phénoménologie de Lambert à celle de Pierce, en passant par Hegel, Brentano, Husserl etc. ? Et, si c'est le cas, lorsqu'on y trouve un usage du concept d'horizon, est-il articulé au même problème historico-philosophique du dépassement de l'ontologie (ou d'une certaine ontologie) heideggerienne, et d'une recherche de l'originaire plus ultra?

Or il est essentiel de remarquer que le modèle de l'herméneutique phénoménologique, et l'histoire de l'horizon qui lui appartient, permet non seulement de poser légitimement la question mais constitue également l'instrument le plus adapté pour y répondre. Car c'est en montrant que ces phénoménologies participent, ou non, de cette recherche que, a contrario, l'on pourra déterminer, ou non, des types de phénoménologie, et d'histoire de l'horizon, alternatifs à ceux de l'herméneutique phénoménologique. 
Autrement dit, c'est le principe d'une histoire différenciée des phénoménologies et de l'horizon qui est par là à la fois anticipé et méthodologiquement préparé. Et cette question des phénoménologies se pose évidemment particulièrement pour Husserl, qui constitue la source d'inspiration de l'usage phénoménologique de l'horizon au $\mathrm{Xx}^{\mathrm{e}}$ siècle.

Quant à savoir dans quelle mesure l'on peut dire que, paradoxalement, la phénoménologie husserlienne n'est pas seulement la source d'influence des tenants principaux de l'herméneutique phénoménologique, mais constitue également une phénoménologie alternative à celle-ci, impliquant une autre histoire et un autre problème de l'horizon, déconnecté du motif du dépassement de l'horizon subjectiviste de la métaphysique de la subjectivité, voilà qui ne manquerait pas d'intérêt historique et philosophique pour celle ou celui qui voudrait fixer pour elle, lui, et pour les autres, le type très spécifique de phénoménologie que représente celle de Husserl, le rôle et le problème très particuliers qui y sont assignés à l'horizon, et de se préparer à les employer comme des instruments philosophiques vivants, alternatifs - reléguant au magasin des accessoires l'image que l'on se plaît parfois à donner de Husserl, celui de l'inventeur d'une phénoménologie dont les supposés présupposés intenables devraient être corrigés ou dépassés (Derrida I967b: 2-3), ou qu'il ne s'agirait de lire que pour mieux «prendre mesure de Heidegger» (Franck I98 I : I3).

\section{Bibliographie}

Blumenberg, Hans. Theorie der Lebenswelt, Hrsg v. Manfred Sommer, Berlin, Suhrkamp 20 Iо.

Deniau, Guy. «Fusion des horizons », in C. Berner et D. Thouard (dir.), L'interprétation. Un dictionnaire herméneutique, Paris, Vrin, 2015, p. 216-222.

Derrida, Jacques. L'écriture et la différence, Paris, Éditions du Seuil, $1967 a$.

- - I967b. La voix et le phénomène. Introduction au problème du signe dans la phénoménologie de Husserl, Paris, PUF, I993.

Djian, Aurélien et Claudio Majolino. "What 'Phenomenon' for Hermeneutics? Remarks on the Hermeneutical Vocation of Phenomenology ", in Paul Fairfield, Saulius Geniusas (eds.), Hermeneutics and Phenomenology: Figures and Themes, London, Oxford, NY, Bloomsbury, 2018.

Elm, Ralf. "Heideggers Horizonte» in Ralf Elm (dir.), Horizonte des Horizontbegriffs. Hermeneutische, phänomenologische und interkulturelle Studien, Sankt Augustin, Academia Verlag, 2004, p. I63-190.Franck, Didier. 198I. Chair et corps. Sur la phénoménologie de Husserl, Paris, Les Éditions de Minuit, I993.

Geniusas, Saulius. The Origin of the Horizon in Husserl's Phenomenology, Dordrecht, Heidelberg, New York, London, Springer, $20 \mathrm{I} 2$.

Grondin, Jean. 2005. "La fusion des horizons. La version gadamérienne de l'adæquatio rei et intellectus?" in Archives de Philosophie, tome 68, 2005/3, p. 4OI-4I 8 .

Heidegger, Martin. SZ. Sein und Zeit, Tübingen, Max Niemeyer Verlag, I967 (Être et Temps. Traduction par Emmanuel Martineau, 1985). 
—. GL. Gelassenheit. Pfullingen: Günther Neske I959 («Sérénité » et «Pour servir de commentaire à Sérénité » in Questions III et IV. Trad. fr. J. Beaufret, Paris, Gallimard 20I I, p. I33-I 82).

Held, Klaus. Phänomenologie der politischen Welt, Frankfurt am Main, Peter Lang, 2010.

Henry, Michel. EM. L'essence de la manifestation, Paris, PUF, $20 \mathrm{II}$.

Iser, Wolfgang. 1976. Der Akt des Lesens. Theorie ästhetischer Wirkung, Stuttgart, Fink/UTB, I984.

Jauss, Hans-Robert. I967. "Literaturgeschichte als Provokation der Literaturwissenschaft » in Literaturgeschichte als Provokation, Frankfurt a.M, Suhrkamp, I970, p. I44-208.

Levinas, Emmanuel. TI. Totalité et Infini: Essai sur l'extériorité, Paris, Le Livre de poche, I990.

Marion, Jean-Luc. ED. Étant donné. Essai d'une phénoménologie de la donation, Paris, PUF, 20I3.

— —On the Gift: A Discussion between Jacques Derrida and Jean-Luc Marion (Sur le don: une discussion entre Jacques Derrida et Jean-Luc-Marion, traduit de l'américain par Sophie-Jan Arrien)» in God, the Gift and Postmodernism. John D. Caputo and Michael J. Scanlon (dir.), Bloomington and Indianapolis, Indiana University Press I999, p. 54-78).

Merleau-Ponty, Maurice. I945. Phénoménologie de la perception, Paris, Gallimard, 2016.

Ricœur, Paul. I986. "Husserl (I859-I938)" in À l'école de la phénoménologie, Paris, Vrin I998, p. 7-г 8.

Romano, Claude. Au cœur de la raison, la phénoménologie, Paris, Gallimard, 20 го. Schweidler, Walter. 2004. "Hinter dem Horizont. Überlegungen im Anschluss an Merleau-Ponty» in Ralf Elm (dir.), Horizonte des Horizontbegriffs. Hermeneutische, phänomenologische und interkulturelle Studien, Sankt Augustin, Academia Verlag, p. 205-226. 